\title{
Corrigendum: A standardized protocol for repeated social defeat stress in mice
}

Sam A Golden, Herbert E Covington III, Olivier Berton \& Scott J Russo

Nat. Protoc. 6, 1183-1191 (2011); doi:10.1038/nprot.2011.361; published online 21 July 2011; corrected after print 17 December 2014

In the version of this article initially published, there was some confusion over the interpretation of the sentence "Further, defeats should be run under constant veterinary evaluation and with full approval of all necessary institutional review boards and standards." For added clarity, the sentence was changed to read "Further, defeats should be run with full approval of all necessary institutional review boards and standards." The error has been corrected in the HTML and PDF versions of the article.

\section{Corrigendum: Solid-phase proximity ligation assays for individual or parallel protein analyses with readout via real-time PCR or sequencing}

Rachel Yuan Nong, Di Wu, Junhong Yan, Maria Hammond, Gucci Jijuan Gu, Masood Kamali-Moghaddam, Ulf Landegren \& Spyros Darmanis

Nat. Protoc. 8, 1234-1248 (2013); doi:10.1038/nprot.2013.070; published online 30 May 2013; corrected after print 16 January 2015

In the version of this article initially published, the PCR table at Step 34C(iii) was incorrect and the sliding splint sequence in Supplementary Table 3 was also incorrect. The errors have been corrected in the HTML and PDF versions of the article and in the revised Supplementary Table 3.

\section{Corrigendum: Using TRIP for genome-wide position effect analysis in cultured cells}

Waseem Akhtar, Alexey V Pindyurin, Johann de Jong, Ludo Pagie, Jelle ten Hoeve, Anton Berns, Lodewyk F A Wessels, Bas van Steensel \& Maarten van Lohuizen

Nat. Protoc. 9, 1255-1281 (2014); doi:10.1038/nprot.2014.072; published online 8 May 2014; corrected after print 16 January 2015

In the version of this article initially published, the final concentration of tamoxifen used in Step 53 of the Procedure was listed as $1 \mathrm{mM}$; it should be $1 \mu \mathrm{M}$. The error has been corrected in the HTML and PDF versions of the article.

\section{Corrigendum: Functional genomics platform for pooled screening and generation of mammalian genetic interaction maps}

Martin Kampmann, Michael C Bassik \& Jonathan S Weissman

Nat. Protoc. 9, 1825-1847 (2014); doi:10.1038/nprot.2014.103; published online 3 July 2014; corrected after print 16 January 2015

In the version of this article initially published, a sentence in Step 49 read "Order the top and bottom oligonucleotides corresponding to hit shRNA sequences formatted as in the example below for the target site "TTCTTACTCACCCTAAGAACT". The sentence has been corrected to replace 'target site' with 'guide sequence'. The error has been corrected in the HTML and PDF versions of the article. 
ERRATA AND CORRIGENDA

\section{Erratum: Antibody structural modeling with prediction of immunoglobulin structure (PIGS)}

Paolo Marcatili, Pier Paolo Olimpieri, Anna Chailyan \& Anna Tramontano

Nat. Protoc. 9, 2771-2783 (2014); doi:10.1038/nprot.2014.189; published online 6 November 2014; corrected online 21 November 2014

In the version of this article initially published online, the title was incorrect and was changed to read as follows: 'Antibody modeling using the Prediction of ImmunoGlobulin Structure (PIGS) web server'. The error has been corrected for the PDF and HTML versions of this article. 